\title{
Structural and electronic properties of small neutral $(\mathrm{MgO})_{n}$ clusters
}

\author{
E. de la Puente and A. Aguado \\ Departamento de Física Teórica, Facultad de Ciencias, Universidad de Valladolid, 47011 Valladolid, Spain \\ A. Ayuela \\ Institut für Theoretische Physik, Technische Universität Dresden, 01062 Dresden, Germany \\ J. M. López \\ Departamento de Física Teórica, Facultad de Ciencias, Universidad de Valladolid, 47011 Valladolid, Spain
}

(Received 23 April 1997)

\begin{abstract}
$A b$ initio perturbed ion calculations are reported for neutral stoichiometric $(\mathrm{MgO})_{n}(n \leqslant 13)$ clusters. A great number of isomer structures are identified and studied. For the isomers of $(\mathrm{MgO})_{n}(n \leqslant 7)$ clusters, a full geometrical relaxation is considered. Correlation corrections are included for all cluster sizes using the Coulomb-Hartree-Fock model proposed by Clementi [IBM J. Res. Dev. 9, 2 (1965)]. The results obtained compare favorably with the experimental data and other previous theoretical studies. The inclusion of correlation is crucial in order to achieve a good description of these systems. We find a number of important isomers that allow us to interpret the experimental magic numbers without the assumption of structures based on $(\mathrm{MgO})_{3}$ subunits. Finally, as an electronic property, the variations in the cluster ionization potential with the cluster size are studied and related to the structural isomer properties. [S0163-1829(97)00236-1]
\end{abstract}

\section{INTRODUCTION}

Clusters provide a new state of aggregation from which the condensed matter properties eventually emerge, being thus of formidable interest in the investigaton of the transition from the molecular world to solid state physics. Small clusters often present substantial deviations in their physical and chemical properties when compared to both the molecule and the bulk phase. Rationalizing the evolution of those properties with the cluster size is a major challenge to present-day science. As an example, for an intermediate-size cluster, the number of different isomers that may coexist in a small-energy range may be quite large. Though it is a very difficult task, a full characterization of those isomer configurations for each cluster size would be highly desirable for a good understanding of cluster growing processes.

In this paper we focus our interests on small stoichiometric $(\mathrm{MgO})_{n}$ clusters. These have received special attention in the past few years from both experimentalists and theoreticians. Saunders ${ }^{1,2}$ published mass-spectra and collision-induced-fragmentation data for sputtered $\mathrm{MgO}$ cluster ions and found enhanced stabilities for $(\mathrm{MgO})_{n}^{+}$clusters with $n=6,9,12$, and 15 . The results were interpreted there in terms of $(\mathrm{MgO})_{3}$ subunits from which the clusters were built. Ziemann and Castleman ${ }^{3-5}$ performed experimental measurements by using laser-ionization time-of-flight mass spectrometry. 6,7 They found "magic clusters"' at $n=2$, $4,6,9,12$, and 15 . In order to explain the features observed in their mass spectra, they performed theoretical calculations by using the rigid ion and the polarizable ion shell models. ${ }^{8-10}$ Their main conclusion was that the clusters form compact cubic structures similar to pieces of the $\mathrm{MgO}$ crystal lattice. Self-consistent calculations on these clusters were performed by Moukouri and Noguera ${ }^{11,12}$ with the use of a semiempirical tight-binding approach. The necessity of go- ing beyond a fully ionic model such as that of pair potential interactions was noted therein. Recio et al. ${ }^{13,14}$ reported $a b$ initio molecular-orbital calculations on neutral clusters of $\mathrm{MgO}$ containing up to 26 atoms. These calculations included correlation effects in the clusters with $n=1-6$ molecules. Their results were in agreement with Saunders's hypothesis, in the sense that structures based on the $(\mathrm{MgO})_{3}$ subunit were preferred over the cubiclike ones. Very recently, Malliavin and Coudray ${ }^{15}$ performed calculations on $(\mathrm{MgO})_{n}$ $(n \leqslant 6)$ clusters using the DMOL method. The geometries obtained in that work are in total agreement with those presented by Recio et al.

Each particular work from those mentioned above represent, in principle, an improvement over the preceding investigations. Pair potential models provided initial theoretical attempt to identify and classify different isomers and calculate the energy differences between them. Nevertheless, some selected set of empirical parameters for $\mathrm{Mg}^{q+}$ and $\mathrm{O}^{q-}$ $(q=1,2)$ ions has to be used as input and that set is the same for ions that are in nonequivalent cluster sites. The studies by Moukouri and Noguera added self-consistency to the calculations, but they were not free of empirical parameters. Finally, $a b$ initio study on these systems was carried out by Recio et al. Several approximations had to be done in their calculations, however, in order to maintain the computational time at reasonable values: (a) Large geometrical distortions in their considered isomers were not allowed, as the optimization process was carried out just by varying the nearestneighbor distance $R$ (for ringlike structures, the stacking distance between rings was also independently optimized) and, for some structures, even this value was kept fixed; (b) several other isomers, which may be important in a more complete characterization of the different isomer structures adopted by small $(\mathrm{MgO})_{n}$ clusters, were not considered; and (c) correlation corrections could be included (at the MP2 
level) only in clusters containing up to 12 atoms.

The present work undertakes an extensive and systematic $a b$ initio study of $(\mathrm{MgO})_{n}$ clusters with $n$ up to 13 . We have used the $a b$ initio perturbed ion (AIPI) model, ${ }^{16}$ which is based on the theory of electronic separability ${ }^{17,18}$ (TES) and the $a b$ initio model potential approach of Huzinaga et al., ${ }^{19}$ within the restricted Hartree-Fock approximation. The model has been used by our group in several studies on $(\mathrm{NaCl})_{n}$ (Ref. 20) and ( $\mathrm{NaI})_{n}$ (Ref. 21) clusters and also in a global study of alkali-halide clusters. ${ }^{22}$ Although we do not expect to obtain accurate results for the only partially ionic $\mathrm{MgO}$ molecule, our main concern is by far not the molecule, but intermediate-size clusters where a full range of different isomer structures may be studied. The solid $\mathrm{MgO}$ has been described excellently by the AIPI model ${ }^{23}$ and precise results are achieved for $(\mathrm{MgO})_{n}$ clusters from $n=3$ on.

Our calculations represent a major advance with respect to pair potential or semiempirical methods. The ion-cluster consistency achieved in the calculations results in a different $a b$ initio description of each nonequivalent ion in the cluster, without requiring the use of any empirical parameter. ${ }^{20-22}$ On the other hand, they represent an alternative description to the molecular-orbital models and complete the results obtained by Recio et al. in the following aspects: (a) For $(\mathrm{MgO})_{n}(n \leqslant 7)$ clusters, a full geometrical relaxation of the different isomers has been considered, (b) we have studied a more complete set of isomers; and (c) correlation corrections have been included for all cluster sizes (up to 26 atoms). Finally, some electronic properties such as ionization potentials have been also considered.

Results presented here are also aimed at assisting in the interpretation of possible future experimental investigations on these clusters. Renewed interest in isomer geometries has recently emerged because of the drift tube experimental studies, which, by measuring the mobility of cluster ions through an inert buffer gas under the influence of a weak electric field, provide valuable information about the cluster geometries $^{24-26}$. However, we have no knowledge of experimental studies of this kind on $(\mathrm{MgO})_{n}$ clusters.

The rest of the paper is structured as follows. In Sec. II we give a brief resume of the AIPI model for the study of clusters (the interested reader can obtain more details in our previous works ${ }^{20-22}$ ) and some computational details. Section III deals with the principal structural and electronic results of the present study. Conclusions are given in Sec. IV.

\section{COMPUTATIONAL METHOD}

The $a b$ initio perturbed ion model ${ }^{16}$ was originally designed for the description of ionic solids ${ }^{27}$ and subsequently adapted to the study of clusters in our group. ${ }^{20-22}$ Its theoretical foundation lies in the theory of electronic separability $^{28}$ for weakly overlapping groups ${ }^{29}$ and its practical implementation in the Hartree-Fock $(\mathrm{HF})$ version of the TES. ${ }^{17,18}$ The HF equations of the cluster are solved in localized Fock spaces by breaking the cluster wave function into local nearly orthogonal group functions (ionic in nature in our case). When the self-consistent process (see details below) finishes, the outputs are the total cluster energy $E_{c l u s}$ and a set of localized wave functions for each geometrically nonequivalent ion in the cluster. The cluster energy can be written as a sum of ionic additive energies ${ }^{20-22}$

$$
E_{\text {clus }}=\sum_{R=1}^{N} E_{a d d}^{R},
$$

where the sum runs over all ions in the cluster and the contribution of each particular ion to the total cluster energy $\left(E_{\text {add }}^{R}\right)$ can be expressed in turn as a sum of intraionic (net) and interionic contributions

$$
E_{\text {add }}^{R}=E_{n e t}^{R}+\frac{1}{2} \sum_{S(\neq R)} E_{i n t}^{R S}=E_{n e t}^{R}+\frac{1}{2} E_{\text {int }}^{R} .
$$

Once $E_{\text {clus }}$ has been obtained, the binding energy per molecule of the $(\mathrm{MgO})_{n}$ cluster with respect to the dissociation process $(\mathrm{MgO})_{n} \rightarrow n \mathrm{Mg}^{2+}+n \mathrm{O}^{-}+n e^{-}+n E_{\text {bind }}$ is given by

$$
E_{\text {bind }}=-\frac{1}{n}\left[E_{\text {clus }}-n E_{0}\left(\mathrm{Mg}^{2+}\right)-n E_{0}\left(\mathrm{O}^{-}\right)\right],
$$

where $E_{0}\left(\mathrm{Mg}^{2+}\right)$ and $E_{0}\left(\mathrm{O}^{-}\right)$are the energies of the $\mathrm{Mg}^{2+}$ and $\mathrm{O}^{-}$free ions, respectively.

The localized nature of the AIPI procedure has some advantages over the usual molecular-orbital models. As in weakly overlapping systems, the correlation energy correction is almost intraionic in nature (being therefore a sum of contributions from each ion), the localized cluster-consistent ionic wave functions may be used to attain good estimations of this correction. In this paper, the correlation energy correction is obtained through Clementi's Coulomb-HartreeFock method. ${ }^{30,31}$ In addition, it also allows the development of computationally efficient $\operatorname{codes}^{32}$ that make use of the large multi- $\zeta$ basis sets of Clementi and Roetti ${ }^{33}$ for the description of the ions. In this respect, our optimizations have been performed using basis sets $5 s 4 p$ for $\mathrm{Mg}^{2+}$ and $6 s 4 p$ for $\mathrm{O}^{2-}$, respectively. Inclusion of diffuse basis functions has been checked and shown to be unnecessary. A general discussion of the election of the most appropriate basis set within the AIPI model has been given elsewhere. ${ }^{22}$

A few comments regarding the weak-overlap assumption and the description of the $\mathrm{O}^{2-}$ anions are worth mentioning. $\mathrm{O}^{2-}$ is an unstable anion in free space, so a basic problem in an ionic description of $\mathrm{MgO}$ clusters is the correct description of the $\mathrm{O}^{2-}$ wave function. Luaña et al. have shown how the $\mathrm{O}^{2-}$ anion is stabilized by the action of the crystal environment in solid $\mathrm{MgO} .{ }^{23}$ Specifically, the $2 p$ oxygen orbital experiences a large contraction in the lattice, which makes it stable as an embedded anion. The resulting $\mathrm{O}^{2-}$ wave function leads to a diamagnetic susceptibility and an electron kinetic-energy increase in quantitative agreement with the experiment. Thus the quality of that wave function is asserted. An analysis of the perturbed ion (PI) results reveals that the orbital contraction is mainly due to the action of the projection operators. The same conclusion has been achieved for the $\mathrm{MgO}$ clusters studied here. The projection operator supplies two major effects. ${ }^{29}$ On the one hand, it tries to maintain the strong-orthogonality hypothesis, being therefore responsible for the orbital contraction. On the other hand, it has a well-defined physical meaning, namely, its expectation value coincides exactly with the overlap energy if we assume that this overlap is "weak." By "weak", it is meant that the products of two overlap integrals $\left(\mathbf{S}_{A B}\right)_{i j}\left(\mathbf{S}_{C D}\right)_{k m}$ with $A$ 
$\neq B$ and $C \neq D$ are negligible $\left[\left(\mathbf{S}_{A B}\right)_{i j}=\left\langle\phi_{i}^{A} \mid \phi_{j}^{B}\right\rangle\right.$, where $\phi_{i}^{A}$ is the $i$ th orbital of the ion $A]$. This corresponds to the following truncation of the Löwdin expansion ${ }^{34}$ for $\mathbf{S}^{-1}$ :

$$
\mathbf{S}^{-1} \approx \mathbf{I}-\mathbf{T} \quad(\mathbf{T}=\mathbf{S}-\mathbf{I}) .
$$

Within this context, the hypothesis of weakly overlapping ions does not imply that the overlap contribution to the cluster energy is negligible. What is neglected is those contributions to the overlap energy coming from second and higher orders in the Löwdin expansion. The overlap energies of the $\mathrm{O}^{2-}$ anions in the $(\mathrm{MgO})_{3}$ clusters are just $\sim 0.5 \mathrm{eV}$ larger than the corresponding value in the solid $(12.84 \mathrm{eV}$ for $\left.\mathrm{O}^{2-}: \mathrm{MgO}\right)$ and they are decreasing functions of the cluster size. The assumption of weakly overlapping ions is good in the solid (the results are exceptional) and we consider that such a small increase in the overlap energies does not invalidate that assumption for cluster studies. Our basis set for the oxygen anion is flexible enough because by adding diffuse basis functions the cluster energy does not lower anymore and the projection operator is not more effective in projecting the frozen orbitals out of the active space. [We would like to remark that an extensive set of different basis functions was considered in test calculations on $(\mathrm{MgO})_{2}$ and $(\mathrm{MgO})_{6}$ isomers. The ones selected gave the lowest cluster energies and achieved saturation in the value of the overlap energies.]

We have used the following self-consistent method. for a given distribution of the ions forming the cluster, we consider one of them as the active ion $R$ (for instance, a particular oxygen anion) and solve the self-consistent-field equations for anion $R$ in the field of the remaining ions, which are considered frozen at this stage. The solution obtained is transferred to all the anions equivalent to anion $R$, that is, to the anions that have equivalent positions in the cluster. Next, we take a nonequivalent oxygen anion (anion $S$ ) as the active ion and repeat the same process. Evidently, since anions $S$ are not equivalent to anions $R$, the energy eigenvalues and wave functions of electrons in anions $S$ are different from those of anions $R$. We continue this process in the same way until all the nonequivalent anions have been exhausted. The same procedure is then followed for the magnesium cations. The process just described is a PI cycle. We iterate the PI cycles until convergence in the total energy of the cluster is achieved.

As input geometries we considered cubic structures resembling pieces of bulk $\mathrm{MgO}$ crystal, ring structures (mainly hexagonal, but also some octagonal and decagonal), mixed structures made up usually of a regular isomer with a molecule attached to it, and some more open structures such as the truncated octahedron for $n=12$ or the wurtzite piece for $n=7$. These trial geometries have been either taken from the geometries considered in pair potential calculations on $\mathrm{MgO}$ and alkali-halide clusters or intuitively guessed with the experience gathered in our previous works on alkali-halide clusters. The input geometries have been fully relaxed (that is, the total cluster energy has been minimized with respect to variations in all the $3 N-6$ independent variables, where $N$ is the number of ions) for $(\mathrm{MgO})_{n}(n \leqslant 7)$ clusters at the HF level. A simplex downhill algorithm has been used in these calculations. ${ }^{35,36}$ For clusters with $n=8-13$ this proce- dure became computationally quite expensive and we turned to geometrical optimizations (also at the HF level) with respect to a limited number of relevant parameters. Cuboid structures have been relaxed with respect to a single parameter, the first-neighbor cation-anion distance, whereas in the ring structures the cation and anion distances to the center of the ring and the distance between rings have been varied independently. For the mixed structures, the part of the regular structure that is nearest (first and second neighbors) to the attached molecule is relaxed together with the molecule and the rest of the isomer is kept fixed at the geometrical configuration with the minimum energy found for that isomer without the molecule attached. The Coulomb-Hartree-Fock (CHF) correlation correction is then introduced for all the studied isomers at the equilibrium geometries found at the HF level. These geometries are further scaled (without shape modifications) until the minimum CHF energy is achieved. We do not expect, from the results obtained by Recio and Pandey, ${ }^{13}$ that inclusion of correlation substantially modifies the isomers shape. We have carried out these scaling calculations mainly because they can affect the ringlike and cubiclike structures differently (and therefore the energetic isomer ordering) and also in order to assess its influence on the averaged cluster bond length and its evolution towards the bulk value.

\section{RESULTS}

\section{A. Lowest-energy structures and isomers}

Results concerning the structural properties of small neutral $(\mathrm{MgO})_{n}$ clusters, obtained by following the method explained in the preceding section, are shown in Fig. 1. Small and large spheres are used to represent $\mathrm{Mg}$ and $\mathrm{O}$ ions, respectively. The most stable CHF structure (first isomer) is shown on the left-hand side for each cluster size. The rest of the structures are the low-lying isomers obtained in the calculation. For clusters with three, four, and five molecules, we show two different views of some isomers. The numbers given below each isomer are the total cluster energy differences with respect to the ground state (denoted GS in the figure) at the CHF (first row) and HF (second row) levels.

For small cluster sizes $(n=2-6)$ only two isomers have been considered. The ground-state geometries in the HF calculations are mainly rings: hexagonal for $n=3$, decagonal for $n=5$, and a stacking of two hexagonal rings for $n=6$. However, for $n=4$ the GS is a cube. The inclusion of correlation does not change the GS structures except for $n=5$, where a distorted cube with a $\mathrm{MgO}$ molecule attached to it becomes energetically more stable. These results are in total agreement with the results of Recio et al. ${ }^{14}$ and Malliavin and Coudray. ${ }^{15}$ However, the isomers obtained in our calculations have large geometrical distortions compared to those obtained by Recio et al., mainly those that have nonringlike configurations: The first excited isomer for $n=3$ was obtained after a full relaxation of a planar piece of the $\mathrm{MgO}$ bulk lattice; this planar piece, with no distortions, is also the first excited isomer in the Recio et al. results, but we can see in Fig. 1 that the final geometry is greatly distorted with respect to the perfect bulk structure. This kind of deformation has been obtained also in lithium halide ${ }^{22}$ and sodium iodide $^{21}$ clusters. The above results show that the PI calcula- 

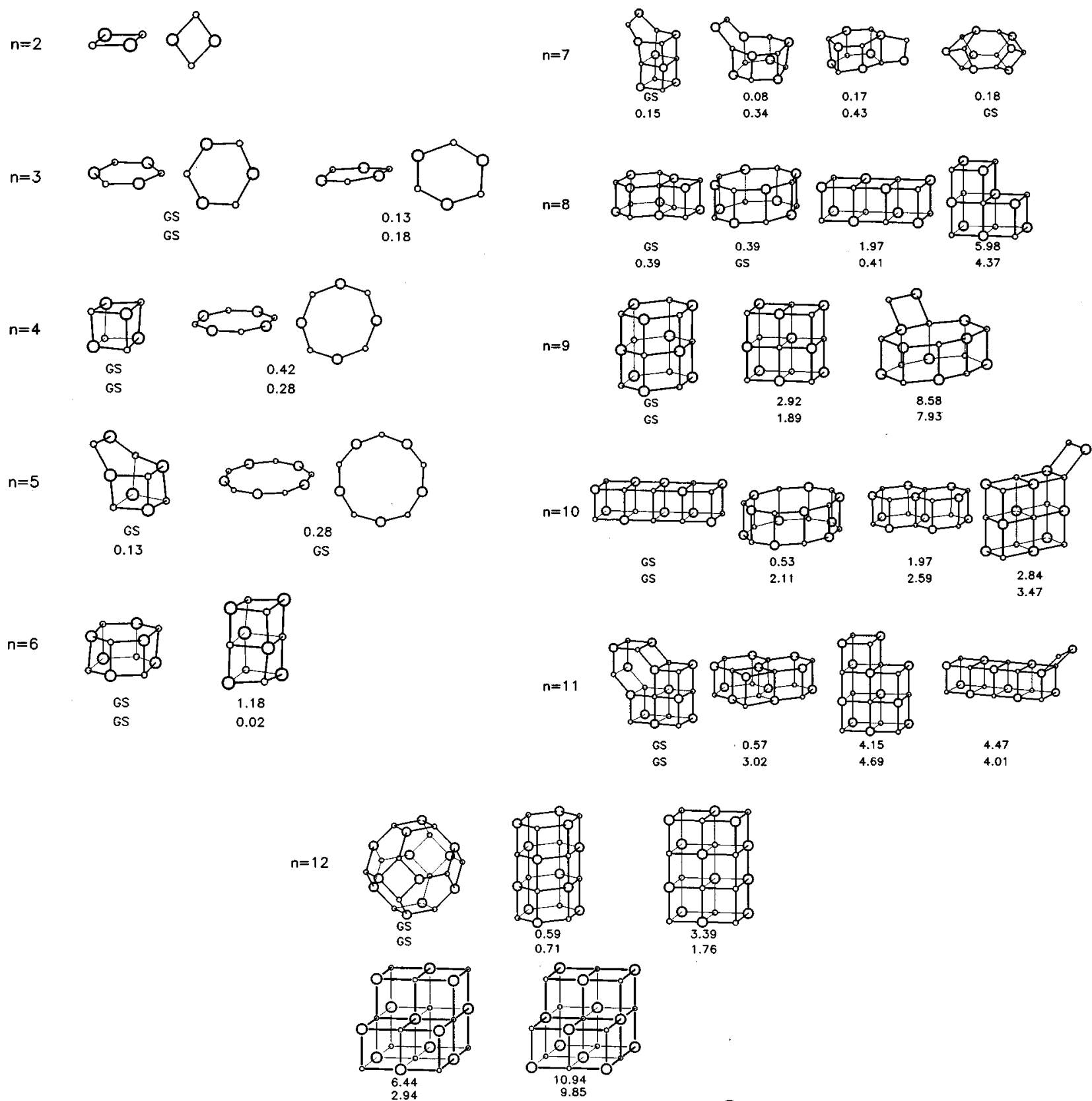

$n=13$
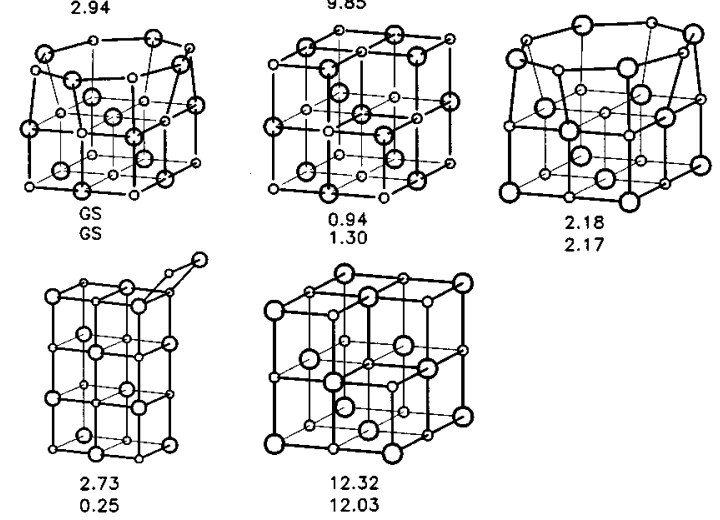

FIG. 1. Isomer geometries for $(\mathrm{MgO})_{n}$ clusters. The most stable CHF structure (first isomer) is shown on the left-hand side. Total energy differences (in $\mathrm{eV}$ ) with respect to the most stable structure are given for each isomer (first row, $\mathrm{CHF}$; second row, $\mathrm{HF}$ ). $\mathrm{Mg}^{2+}$, small spheres; $\mathrm{O}^{2-}$, large spheres. For $n=3-5$, two different views of some isomers are provided. 
tions agree with those of other more "popular" ab initio calculations: GAUSSIAN (Refs. 13 and 14) and DMOL. ${ }^{15}$ That means that the PI method is a good candidate for obtaining the ground state and other geometrical and electronic properties of clusters with ionic bonding such as the $(\mathrm{MgO})_{n}$ clusters considered in this paper.

For the clusters with $n=7-13$ we have considered a larger number of initial geometries in our calculations. Overall, the ground-state geometries obtained in the HF calculations are the same as those obtained by Recio et al.. We appreciate differences only for those cluster sizes in which the ground-state structures that we obtain were not considered by Recio et al. in their calculations $(n=11-13)$.

The inclusion of correlation in the calculations changes the GS geometries for $n=7$ and 8 . These results cannot be compared with the calculations performed by Recio et al. because they did not include the correlation contribution in this range of sizes or with the calculations of Malliavin and Coudray because they did not perform any calculations in this size range. For $n=7$ the HF ground-state isomer is the wurtzite piece, whereas the CHF ground state is the bulklike $(\mathrm{MgO})_{6}$ piece with a $\mathrm{MgO}$ molecule attached to it. For $n=8$ the HF GS is a stacking of two octagonal rings, whereas the CHF GS is a mixed structure formed by "joining" the most stable $(\mathrm{MgO})_{6}$ and $(\mathrm{MgO})_{4}$ isomers. The last structure is also the second isomer in the HF results. For $n=9$ the ground-state isomer is a stacking of three hexagonal rings, the same as in the HF calculations; the other isomers considered are a piece of $\mathrm{MgO}$ bulk and the $(\mathrm{MgO})_{8} \mathrm{HF}$ GS with a molecule attached to it. For $n=10$ the GS geometry is a piece of the $\mathrm{MgO}$ lattice. The other isomers considered in our calculations are, in order of increasing energy, a stacking of two decagonal rings, a piece formed by joining two stackings of two hexagonal rings by a face, and a piece of the bulk $\mathrm{MgO}$ lattice with a molecule attached to it [attaching a molecule to the $(\mathrm{MgO})_{9} \mathrm{GS}$ leads to an isomer that lies higher in energy]. For $n=11$ the GS isomer is a mixed structure composed by a stacking of two hexagonal rings plus a bulklike part and the other isomers considered are either from mixed type or bulklike. A truncated octahedron is obtained as the GS for $n=12$. This isomer has been found also to be especially stable in $(\mathrm{NaI})_{12}$ clusters. ${ }^{21}$ It was not considered in Ref. 14. Finally, a mixed structure composed of a piece of the $\mathrm{MgO}$ lattice and an octagon is found as the $(\mathrm{MgO})_{13}$ ground state. Our second isomer is the defect-cuboid structure, which was the single isomer tried in Ref. 14. A point that is worth mentioning from these two final cluster sizes is that there is the possibility to obtain different isomers with the same geometrical shape, but with cations and anions interchanged. The electrostatic interaction tends to stabilize energetically anions, ${ }^{20,21}$ so those isomers with a central oxygen are more stable.

We compare now the preceding results with the results obtained with the PI model for alkali-halide $(A X)_{n}$ clusters. ${ }^{20-22}$ In our previous works we found evidence of a clear-cut separation between the structural properties of different alkali-halide clusters. $(\mathrm{KX})_{n}$ and $(\mathrm{Rb} X)_{n}$ clusters showed almost from the very beginning a preference to adopt minimum-energy structures that are fragments of the bulk lattice. However, $(\mathrm{LiX})_{n}$ clusters adopted preferentially more open structures. $(\mathrm{NaX})_{n}$ showed an intermediate behavior.
Our structural results for $(\mathrm{MgO})_{n}$ clusters show, in outline, many similarities to those of $(\mathrm{Li} X)_{n}$ or $(\mathrm{Na} I)_{n}$ clusters. These are the least ionic compounds in the alkali-halide series. Rounded geometries had been proposed by Twu et al. ${ }^{7}$ in those cases showing a decreased ionic bonding character. Then, our minimum-energy isomers are consistent with a reduced ionic character in $(\mathrm{MgO})_{n}$ clusters. This nonfully ionic character was also pointed out in the analysis of Recio et al. ${ }^{14}$ The discussion there was based on a comparison with the classical results of Ziemann and Castleman, ${ }^{3}$ who performed pair potential calculations using two different (but both fully ionic) models, with different nominal charges assigned to the ions. The calculations for $\left(\mathrm{Mg}^{+1} \mathrm{O}^{-1}\right)_{n}$ clusters revealed mainly cubic structures, whereas in $\left(\mathrm{Mg}^{+2} \mathrm{O}^{-2}\right)_{n}$ calculations a set of much more open structures was obtained. As the $\left(\mathrm{Mg}^{+1} \mathrm{O}^{-1}\right)_{n}$ calculations explained better the experimental mass spectra, Ziemann and Castleman concluded that pieces of the $\mathrm{MgO}$ bulk lattice are obtained for small $(\mathrm{MgO})_{n}$ clusters. Both our calculations and those of Refs. 14 and 15 obtain results that are between these two limit cases, so nominal charges between $|1|$ and $|2|$ should be ascribed to $\mathrm{Mg} / \mathrm{O}$ ions in classical pair potential simulations. However, our calculations show that a description in terms of weakly overlapping $\mathrm{Mg}^{2+}$ and $\mathrm{O}^{2-}$ groups is completely appropriate provided that all the relevant quantummechanical interactions (overlap included) are accounted for properly and a full ion-cluster consistency is achieved. It is worth stressing that such a description is not in conflict with a possible assignment of fractional charges to each ion. A population analysis is best carried out directly in terms of the total density, following Bader's scheme for deriving atomic properties from the topology of the charge density. ${ }^{37}$ That has been done recently in PI calculations on solids ${ }^{38}$ and fractional ionic charges have been derived for a variety of ionic crystals.

From the study of small $(\mathrm{MgO})_{n}(n=2-13)$ clusters we conclude that, in the earliest stages of cluster growth, the bulk lattice fragments are not the GS isomers except for some particular values of $n$. For the rest of cluster sizes other (more open) structures are preferred. Thus the convergence to the bulk structure is not reached yet in this size range (the solid $\mathrm{MgO}$ crystallizes in the fcc structure ${ }^{39}$ ).

We can appreciate in Fig. 1 for $n=6,9$, and 12 (and also for other cluster sizes) that the inclusion of correlation enhances the stability of the hexagonal isomers over the cubic ones. Though in the size range covered open structures are preferred over the cubic ones, eventually there will be a cluster size where the cubic structures become energetically more stable and this specific value of $n$ will depend on the inclusion or exclusion of correlation in the calculations. The above observation suggests that this "critical" $n$ will be larger upon inclusion of correlation, that is, pieces of the $\mathrm{MgO}$ bulk lattice will appear as GS isomers for values of $n$ that are larger in CHF calculations than in HF calculations.

The magic numbers for small $(\mathrm{MgO})_{n}^{+}$clusters emerging from mass spectra experiments have been explained by Saunders ${ }^{1}$ and by Recio et al. ${ }^{14}$ as resulting from specially enhanced structures based on the stacking of $(\mathrm{MgO})_{3}$ subunits. Our results show that, at least for neutral $(\mathrm{MgO})_{n}$ clusters [and also for ionized $(\mathrm{MgO})_{n}^{+}$clusters if the vertical 


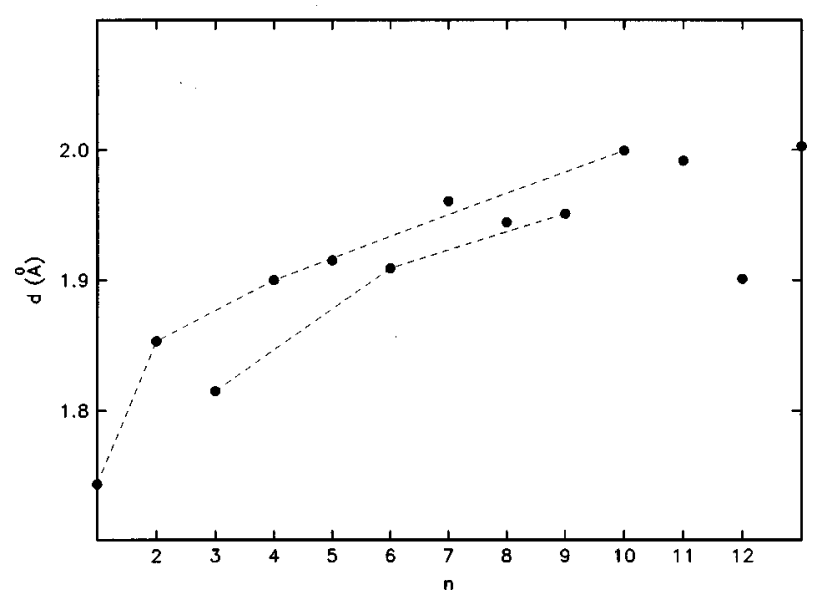

FIG. 2. Averaged nearest-neighbor $\mathrm{Mg}-\mathrm{O}$ distance for the $\mathrm{CHF}$ ground-state structures of Fig 2. The two lines join cubic and hexagonal clusters.

approximation holds $\left.{ }^{14}\right]$, this conclusion is not universal. The truncated octahedron obtained as the ground-state isomer for $n=12$ in our calculations indicates that this interpretation should be taken with some caution.

\section{B. Interionic distances and binding energies}

In Fig. 2 we present the average interionic CHF distance $d$ between $\mathrm{Mg}$ and $\mathrm{O}$ nearest neighbors for the ground-state geometry as a function of the cluster size $n$. $d$ presents an irregular behavior as a function of $n$ due to the fact that the ground-state geometries for the different values of $n$ do not correspond to the same type of structural family. The points that correspond to the same structural family have been joined by the two dashed lines and it can be appreciated that the variation of $d$ is smoother for each structural family. The value of $d$ increases with the number of ions in the cluster. The cubic clusters have an interatomic distance larger than the hexagonal ones. The isomers that resemble pieces of the bulk $\mathrm{MgO}$ lattice are the important set in a comparison with the bulk value. In the $\mathrm{MgO}$ crystal $d=2.106 \AA$ (Ref. 39) and we obtain a value of $d=1.999 \AA$ for the GS isomer with $n=10$. The saturation value is not achieved yet, but the trend is the correct one. The contraction of the distances induced by the inclusion of correlation is more important in cubiclike pieces than in ringlike ones: The average effect in cubic structures is a contraction of approximately $4 \%$, while in hexagonal structures the contraction is only of some $2 \%$. The inclusion of correlation is necessary in order to obtain quantitative agreement with the experimental bulk value in the limit of $n$ large.

In Fig. 3, we represent $E_{\text {bind }}$ as a function of $n$ for both the HF and CHF calculations. The general trend is an increase of the binding energy with $n$ in both cases. Superposed to that general trend, especially stable clusters are predicted for certain values of $n$ ("magic numbers"); these are identified in the figures as maxima or pronounced changes in the slope. We observe that both types of calculations do not predict the same set of magic numbers. The HF curve is quite smooth for small values of $n$ and maxima are observed only at $n=9,12$ (pronounced slope changes are not observed). The inclusion of correlation effects (CHF curve) is

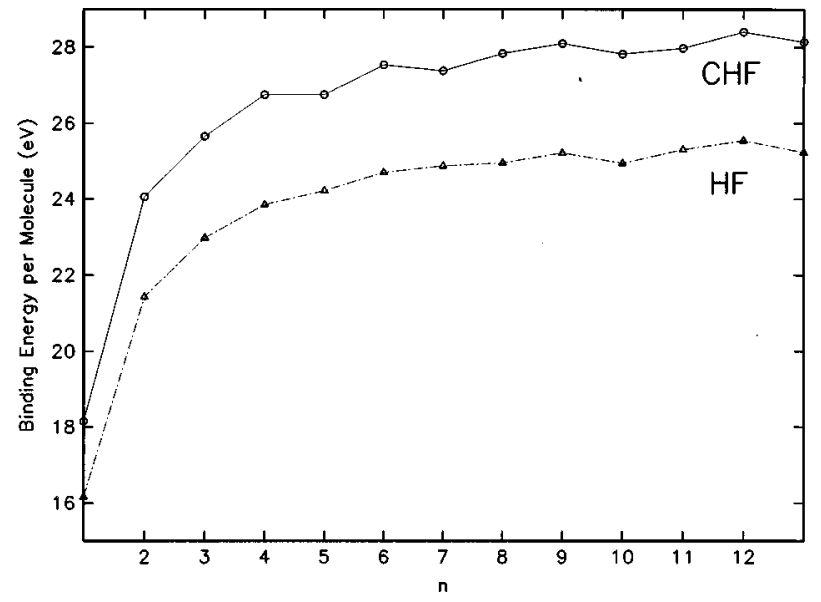

FIG. 3. Binding energy per molecule as a function of the cluster size.

crucial to achieve agreement with the experimental results, ${ }^{1,3}$ which predict magic clusters at $n=2,4,6,9,12,15, \ldots$ The maxima at $n=6,9,12$ are clear and a pronounced slope change is appreciable at $n=4$. The abundance maximum obtained experimentally at $n=2$ should probably be related to the singly ionized stoichiometric clusters and not to the neutral clusters. ${ }^{14}$

\section{Cluster ionization potentials}

In this subsection we analyze the variation of the cluster ionization potential (IP) with the cluster size. The output of the PI calculation contains a fully cluster-consistent wave function for each nonequivalent ion in the cluster. In our model, the electron must be extracted from a specific localized anionic orbital when the cluster is ionized. The vertical IP of the cluster is calculated as the smallest binding energy of a $2 p$ anionic electron (Koopmans's approximation). The results are given in Fig. 4, where we have plotted the eigenvalues of the $2 p$ orbitals of $\mathrm{O}^{2-}$ anions as a function of $n$ for the most stable structure of each cluster size in the HF [Fig. 4(a)] and in the CHF [Fig. 4(b)] calculations. We appreciate a band of eigenvalues for each cluster size because the anions can occupy nonequivalent positions in the cluster. These band gaps are indicative of the different reactivity of each nonequivalent cluster site, they can determine the preferred adsorption sites, and they could be helpful in photoionization spectroscopy studies.

The dashed lines, which join the lowest $2 p$ energy eigenvalues for each cluster size, indicate the variation of the HF [Fig. 4(a)] and CHF [Fig. 4(b)] ionization potentials with the cluster size. The variation of the vertical cluster ionization potential with $n$ is related to the relative stabilities of neutral and singly ionized stoichiometric clusters: ${ }^{14}$ When there is a maximum in the IP for a cluster size $n$, the ionized structure is less stable than the neutral one against loss of a $\mathrm{MgO}$ monomer; the opposite occurs at the minima in the IP curve. For the HF results, a set of minima at $n=4,7$, and 11 and maxima at $n=3,5,8$, and 10 is observed, in agreement with the results of Recio et al. The difference is that they obtain a maximum at $n=12$; but the results are not directly comparable for these cluster sizes $(n=12,13)$ because the isomers that we obtain as the GS were not considered in their calcu- 


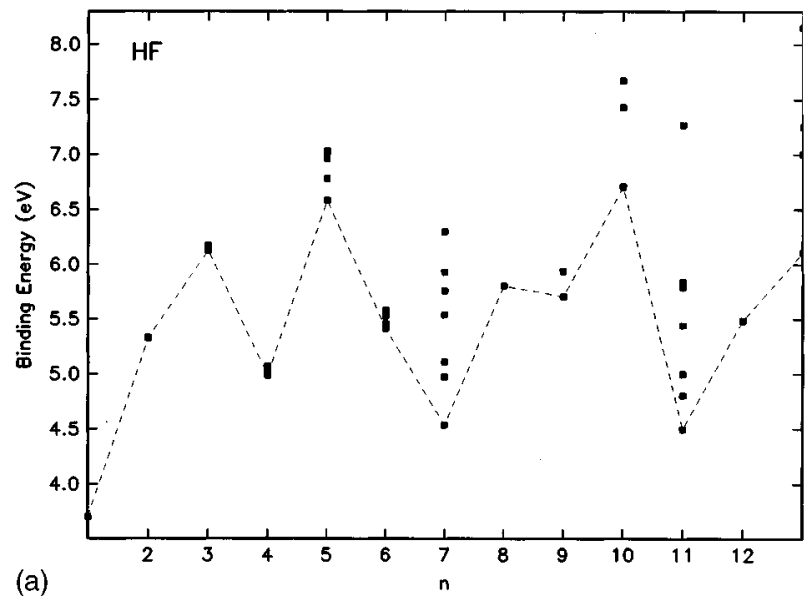

(a)

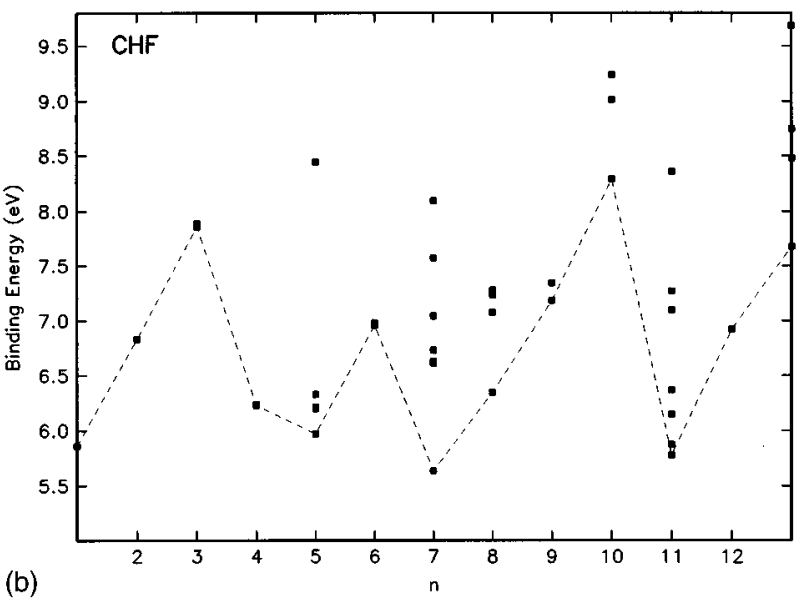

FIG. 4. Orbital energies (with opposite sign) of the $2 p$ levels of $\mathrm{O}^{2-}$ anions as a function of the cluster size for the ground-state structures of $(\mathrm{MgO})_{n}$ clusters. The dashed line joins the vertical cluster ionization potentials. (a) HF results and (b) CHF results.

lations. This pattern changes upon inclusion of correlation effects (see the CHF curve): Minima are found at sizes $n=$ 5,7 , and 11, and maxima at $n=3,6$, and 10 . These changes are directly related to the change in the GS isomer for $n=5$. We can give a clear geometrical interpretation to the minima in the light of the localized picture provided by the PI model. At the minima ( $n=5,7$, and 11), mixed structures were found as the most stable isomers (Fig. 1). The least bound anionic $p$ electron in those clusters corresponds always to that oxygen with the smallest number of neighboring ions. That specific oxygen is less stabilized than the other oxygens in the cluster because of the reduced electrostatic interaction for those cluster sites. An electron will be removed from it more easily than for the rest of cluster sizes (for which the GS isomer is more "compact"). Such a clear geometrical interpretation of the maxima in the IP's is not apparent for the small-size range covered in this work: The opposite situation (maxima in the IP for those ground states with the highest symmetries) is not always observed. As the main contribution to the anionic binding energy is the Madelung potential and this is the largest for the bulklike structures, we expect maxima to appear precisely in compact cubic structures for larger cluster sizes.

In simple metallic clusters, the variations in the cluster ionization potential are correlated with the magic numbers, indicating that the magic numbers are electronic in nature. ${ }^{40}$ There is not such a definite connection in ionic clusters and the variations observed in the cluster ionization potential are related mainly to structural features. From our discussion, the existence of such a connection between electronic (IP's) and geometrical properties in $(\mathrm{MgO})_{n}$ clusters becomes clear. The question of the interconnection between electronic, energetic, and geometrical cluster properties has been more thoroughly addressed in ionic alkali-halide materials. ${ }^{22}$

\section{CONCLUSION}

$A b$ initio perturbed ion calculations have been carried out in order to investigate the structural, energetic, and some electronic properties of small neutral stoichiometric $(\mathrm{MgO})_{n}$ clusters. The set of isomers studied is more complete than in previous calculations and some of the isomers not considered previously have resulted in being either the ground-state structure or a low-lying isomer for several cluster sizes. Specifically, we have shown that the interpretation of the magic numbers of small $(\mathrm{MgO})_{n}$ clusters in terms of stackings of $(\mathrm{MgO})_{3}$ subunits is not universal because a truncated octahedron is more stable for $n=12$ than the corresponding hexagonal isomer. General structural distortions have been considered for $(\mathrm{MgO})_{n}(n \leqslant 7)$ clusters and these deformations have been shown to be really important in those isomers with lowest symmetries. Correlation corrections have been included for all cluster sizes with the Clementi's CoulombHartree-Fock method. Their inclusion has been very important in the determination of some ground-state isomers and the energy differences between isomers for each cluster size. Specifically, at $n=5,7,8$, and 10 different ground-state structures are obtained after accounting for correlation effects. Whenever a comparison with previous $a b$ initio calculations has been made the agreement has been good.

The evolution of the average interionic distance with the cluster size has been studied. Clusters from the same structural family show a smooth variation of their interionic distances with $n$. The correlation-induced distance contractions are the largest for those isomers that are fragments of the $\mathrm{MgO}$ bulk lattice. Energetically, however, the correlation correction stabilizes more the hexagonal isomers, so the transition towards bulk structures will take place at larger cluster sizes than HF calculations would predict. This result contrasts with the situation encountered in alkali-halide clusters, where the inclusion of correlation induces a larger energetic stabilization of the cuboid isomers. ${ }^{22,41}$ The $(\mathrm{MgO})_{n}$ magic numbers have been identified as maxima or pronounced slope changes in the binding energy versus cluster size curve. They are $n=4,6,9$, and 12 , in agreement with the experimental results $n=2,4,6,9,12,15, \ldots[n=2$ has been considered to be due to a larger enhancement of the singly ionized $(\mathrm{MgO})_{2}^{+}$species]. Inclusion of correlation has also shown to be completely necessary in order to reproduce the correct (experimental) magic numbers.

The interconnection between electronic and structural cluster properties has been studied by considering the variations in the cluster ionization potential with the cluster size $n$. For the minima in the IP, which occur for the lowest symmetry ground-state isomers at $n=5,7$, and 11 , a clear geometrical interpretation can be given: In those isomers, 
there is always an oxygen anion with a number of neighboring ions notably reduced, from which it is relatively easy (compared to the rest of cluster sizes) to remove an electron and create a singly ionized isomer. Such a definite connection has not been found for the maxima in the size range considered in this work. The corresponding opposite situation of maxima in the IP emerging for all those ground-state isomers with the highest symmetries is not observed. The increased classical electrostatic (Madelung) energy of isomers resembling pieces of the $\mathrm{MgO}$ crystal explains the maximum observed at $n=10$. Then maxima for larger cluster sizes should be expected to appear whenever the ground state becomes a compact fragment of the $\mathrm{MgO}$ bulk lattice.

\section{ACKNOWLEDGMENTS}

This work has been supported by DGICYT (Grant No. PB95-D720-C02-01). A. Aguado acknowledges a grant from Junta de Castilla y León.
${ }^{1}$ W. A. Saunders, Phys. Rev. B 37, 6583 (1988).

${ }^{2}$ W. A. Saunders, Z. Phys. D 12, 601 (1989).

${ }^{3}$ P. J. Ziemann and A. W. Castleman, Jr., Z. Phys. D 20, 97 (1991).

${ }^{4}$ P. J. Ziemann and A. W. Castleman, Jr., J. Chem. Phys. 94, 718 (1991).

${ }^{5}$ P. J. Ziemann and A. W. Castleman, Jr., Phys. Rev. B 44, 6488 (1991).

${ }^{6}$ C. W. S. Conover, Y. A. Yang, and L. A. Bloomfield, Phys. Rev. B 38, 3517 (1988).

${ }^{7}$ Y. T. Twu, C. W. S. Conover, Y. A. Yang, and L. Bloomfield, Phys. Rev. B 42, 5306 (1990).

${ }^{8}$ T. P. Martin, Phys. Rep. 95, 168 (1983).

${ }^{9}$ J. Diefenbach and T. P. Martin, Surf. Sci. 156, 234 (1984).

${ }^{10}$ N. G. Phillips, C. W. S. Conover, and L. A. Bloomfield, J. Chem. Phys. 94, 7498 (1991).

${ }^{11}$ S. Moukouri and C. Noguera, Z. Phys. D 24, 71 (1992).

${ }^{12}$ S. Moukouri and C. Noguera, Z. Phys. D 27, 79 (1993).

${ }^{13}$ J. M. Recio and R. Pandey, Phys. Rev. A 47, 2075 (1993).

${ }^{14}$ J. M. Recio, R. Pandey, A. Ayuela, and A. B. Kunz, J. Chem. Phys. 98, 4783 (1993).

${ }^{15}$ M. J. Malliavin and C. Coudray, J. Chem. Phys. 106, 2323 (1997).

${ }^{16}$ V. Luaña and L. Pueyo, Phys. Rev. B 41, 3800 (1990).

${ }^{17}$ S. Huzinaga and A. P. Cantu, J. Chem. Phys. 55, 5543 (1971).

${ }^{18}$ V. Luaña, Ph.D. thesis, Universidad de Oviedo, 1987.

${ }^{19}$ S. Huzinaga, L. Seijo, Z. Barandiarán, and M. Klobukowski, J. Chem. Phys. 86, 2132 (1987).

${ }^{20}$ A. Ayuela, J. M. López, J. A. Alonso, and V. Luaña, Z. Phys. D 26, S213 (1993); Ann. Fis. (Spain) 90, 190 (1994); Physica B 212, 329 (1996).

${ }^{21}$ A. Aguado, A. Ayuela, J. M. López, and J. A. Alonso, J. Phys. Chem. (to be published).

${ }^{22}$ A. Aguado, A. Ayuela, J. M. López, and J.A. Alonso (unpublished).
${ }^{23}$ V. Luaña, J. M. Recio, and L. Pueyo, Phys. Rev. B 42, 1791 (1990).

${ }^{24}$ G. von Helden, M. T. Hsu, P. R. Kemper, and M. T. Bowers, J. Chem. Phys. 95, 3835 (1991).

${ }^{25}$ M. F. Jarrold, J. Phys. Chem. 99, 11 (1995).

${ }^{26}$ M. Maier-Borst, P. Löffler, J. Petry, and D. Kreisle, Z. Phys. D (to be published).

${ }^{27}$ V. Luaña, M. Flórez, E. Francisco, A. Martín Pendás, J. M. Recio, M. Bermejo, and L. Pueyo, in Cluster Models for Surface and Bulk Phenomena, edited by G. Pacchioni, P. S. Bagus, and F. Parmigiani (Plenum, New York, 1992), p. 605

${ }^{28} \mathrm{R}$. McWeeny, Methods of Molecular Quantum Mechanics (Academic, London, 1994).

${ }^{29}$ E. Francisco, A. Martín Pendás, and W. H. Adams, J. Chem. Phys. 97, 6504 (1992).

${ }^{30}$ E. Clementi, IBM J. Res. Dev. 9, 2 (1965).

${ }^{31}$ S. J. Chakravorty and E. Clementi, Phys. Rev. A 39, 2290 (1989).

32 V. Luaña, A. Martín Pendás, J. M. Recio, and E. Francisco, Comput. Phys. Commun. 77, 107 (1993).

${ }^{33}$ E. Clementi and C. Roetti, At. Data Nucl. Data Tables 14, 177 (1974).

${ }^{34}$ P. O. Löwdin, Adv. Phys. 5, 1 (1956).

${ }^{35}$ J. A. Nelder and R. Mead, Comput. J. (UK) 7, 308 (1965).

${ }^{36}$ William H. Press and Saul A. Teukolsky, Comput. Phys. 5, 426 (1991).

${ }^{37}$ R. F. W. Bader, Atoms in Molecules (Oxford University Press, Oxford, 1990).

${ }^{38}$ A. Martín Pendás, A. Costales, and V. Luaña, Phys. Rev. B 55, 4275 (1997).

${ }^{39}$ N. M. Ashcroft and N. D. Mermin, Solid State Physics (Holt, Rinehart and Winston, New York, 1976).

${ }^{40}$ W. A. de Heer, W. D. Knight, M. Y. Chou, and M. L. Cohen, Solid State Phys. 40, 93 (1987).

${ }^{41}$ C. Ochsenfeld and R. Ahlrichs, Ber. Bunsenges. Phys. Chem. 98, 34 (1994). 